\title{
Evaluation des caractéristiques dosimétriques d'un nouveau type de compteur équivalent au tissu destiné à la dosimétrie individuelle neutronique en radioprotection
}

\author{
J.M. BORDY*, J. BARTHE*, B. BOUTRUCHE*, P. SEGUR**
}

(Manuscrit reçu le 19 mai 1993)

RÉSUMÉ Cet article présente l'évaluation des principales caractéristiques du prototype de compteur proportionnel multicellulaire équivalent au tissu étudié dans notre laboratoire pour la dosimétrie individuelle neutronique. Il comporte plusieurs centaines de canaux creusés dans la cathode en regard des fils anodiques. On montre que sa sensibilité est compatible avec une utilisation en radioprotection et que la dimension du volume simulé permet d'appliquer la méthode microdosimétrique. La distribution de la longueur des cordes, obtenue par simulation avec la méthode de Monte-Carlo dans le cas d'une irradiation isotrope, est proche d'une fonction de Dirac centrée sur la valeur moyenne de la distribution. Enfin, un mélange de gaz spécifique contenant de l'argon, du propane, du dioxyde de carbone et de l'azote est proposé pour le remplissage du compteur.

ABSTRACT The main characteristics of the multicellular tissue equivalent proportional counter studied in our laboratory have been evaluated. It is made of several drift regions viz holes drilled into the cathode in front of the anode wires. It is shown that its sensitivity makes it possible to use it in radiation protection. The size of the simulated volume fulfill the microdosimetric assumptions. The chord length distribution, computed by the Monte-Carlo method, is close to a Dirac function corresponding to the average distribution value. Finally, a specific gas mixture made of argon, propane, carbon dioxide and nitrogen is proposed to fill the counter.

\section{Introduction}

La dosimétrie individuelle des champs de rayonnement mixtes (neutronsphotons) en radioprotection est actuellement assurée au moyen de dosimètres à lecture différée dont la réponse est imparfaite. L'évolution de la réglementation et des techniques a incité le service de dosimétrie à étudier un compteur

* Commissariat à l'énergie atomique, Institut de protection et de sûreté nucléaire (IPSN), DPHD/S DOS, BP 6, F 92265 Fontenay-aux-Roses Cedex.

** CNRS, Centre de physique atomique (CPAT), Université P. Sabatier, 118 Route de Narbonne, F 31062 Toulouse Cedex. 
proportionnel équivalent au tissu biologique (CPET). Ce type de détecteur, sensible aux neutrons et aux photons, permet d'obtenir en temps réel la distribution des transferts linéiques d'énergie (TLE) à partir de la mesure de la distribution des dépôts d'énergie dans le compteur. La connaissance du spectre de TLE permet d'estimer les composantes neutroniques et photoniques, de calculer la dose absorbée, l'équivalent de dose et le facteur de qualité moyen [3]. Cet article présente le résultat de l'évaluation théorique des caractéristiques d'un CPET multicellulaire destiné à la dosimétrie individuelle. Les points étudiés sont : la distribution de la longueur des cordes, la sensibilité du compteur, le volume simulé et la composition du gaz de remplissage.

\section{Description du compteur multicellulaire}

En 1955, Harald H. Rossi développait le premier compteur microdosimétrique répondant aux caractéristiques suivantes [19] :

- fonctionnement en régime proportionnel,

- équivalence au tissu biologique,

- simulation d'un site biologique de la taille d'une cellule.

Le volume sensible de ce compteur est sphérique ; le diamètre de la sphère et la pression du gaz sont fonction du volume que l'on désire simuler. La cathode est fabriquée dans un matériau électriquement conducteur, équivalent au tissu biologique mou (A 150). Le renouvellement continu du gaz, lui même également équivalent au tissu, permet de s'affranchir des problèmes liés à sa dégradation.

Les compteurs fabriqués et utilisés aujourd'hui présentent des caractéristiques voisines de celles du compteur de Rossi. En radioprotection, ils sont surtout utilisés pour la dosimétrie de zone. L'extrapolation des caractéristiques géométriques et fonctionnelles des détecteurs existants vers un dosimètre individuel s'avère difficile, voire impossible. En premier lieu, le faible encombrement d'un dosimètre individuel impose une forte réduction du volume de détection, qui, en conservant une forme sphérique ou cylindrique, entraîne une réduction de la sensibilité d'un facteur 10 à 20 . En second lieu, les problèmes de dégradation et de pollution du gaz sont accentués par l'absence de renouvellement [20].

Les compteurs de forme cylindrique ou sphérique ne présentant pas une sensibilité correcte après miniaturisation, nous avons opté pour la forme parallélépipédique. Elle présente divers avantages tels qu'une plus grande facilité d'usinage et une meilleure logeabilité du capteur dans un boîtier lui même parallélépipédique dans l'optique d'un dosimètre individuel de poche.

Le CPET multicellulaire prototype est constitué de deux cathodes, usinées dans un plastique équivalent au tissu et disposées l'une au-dessus de l'autre (Fig. 1). Une nappe de fils anodiques est tendue dans l'espace séparant les 
deux cathodes. Le plan défini par cette nappe est équidistant des deux cathodes. Les dimensions extérieures maximales hors tout retenues pour le compteur sont : $10 \mathrm{~cm} \times 5 \mathrm{~cm} \times 3 \mathrm{~cm}$.

Pour accroître la sensibilité du capteur, on augmente la "surface d'échange cathode-gaz", c'est-à-dire la surface interne de la cathode en contact avec le gaz. Pour cela, chaque cathode est creusée d'un grand nombre de cavités cylindriques, appelées canaux ou cellules.

Comme dans les CPET cylindriques, les particules neutres (photons et neutrons) interagissant dans la cathode libèrent des particules chargées (principalement des électrons et des protons) qui, en pénétrant dans le gaz, en ionisent les atomes et les molécules. Pour éviter la recombinaison des ions et des électrons, et pour guider ces derniers vers l'anode, un champ électrique, appelé champ électrique de dérive, dirigé selon l'axe longitudinal du canal, est créé à l'intérieur de celui-ci par une différence de potentiel, croissante en direction de l'anode. A cet effet, nous utilisons deux types de plastique équivalent au tissu :

- un plastique de faible résistivité (A 150), créant une surface équipotentielle à chaque extrémité du canal,

- un plastique de forte résistivité (de l'ordre du gigaohm par centimètre) jouant le rôle d'un pont de polarisation entre les deux surfaces équipotentielles.

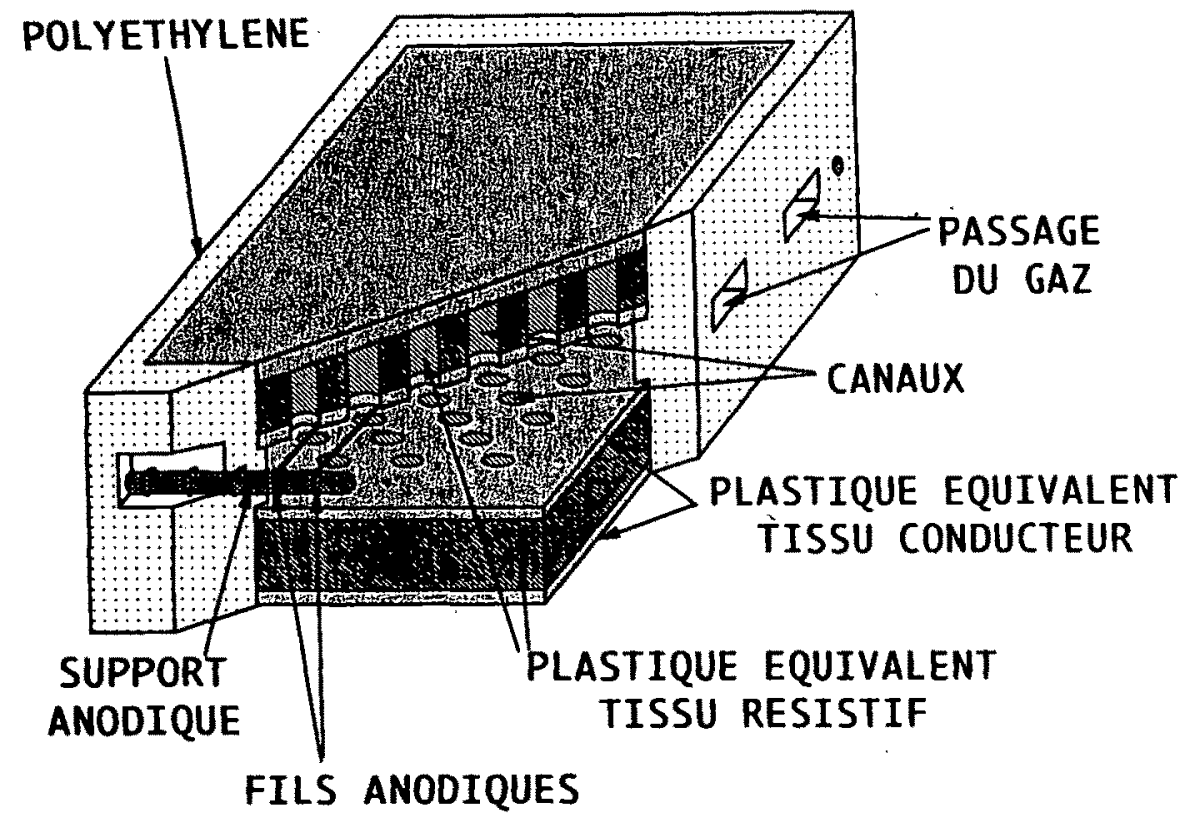

Fig. 1. - Vue en trois dimensions du compteur multicellulaire. $3 D$ view of the multicellular counter. 
Avec une résistivité égale à $1 \mathrm{G} \Omega$. cm et une différence de potentiel de $50 \mathrm{~V}$ appliquée aux extrémités des canaux, la puissance dissipée dans l'ensemble des cathodes est inférieure à $200 \mu \mathrm{W}$ et le courant électrique inférieur à $4 \mu \mathrm{A}$. Cette consommation électrique est parfaitement compatible avec un fonctionnement autonome sur pile ou sur batterie.

\section{Composition du gaz de remplissage}

Le volume du gaz interne au compteur correspond au volume des canaux et de l'espace entre les électrodes et représente le volume sensible. Les particules chargées, issues de l'interaction des neutrons et des photons dans le matériau constituant les cathodes, déposent tout ou partie de leur énergie par ionisation et par excitation des atomes et des molécules du gaz. La composition de ce dernier est équivalente au tissu, de manière à reproduire le plus fidèlement possible le dépôt d'énergie dans le site biologique microscopique simulé. En outre, il est souhaitable d'utiliser un mélange de gaz qui possède un gain élevé pour améliorer la détection des petits événements et pour simplifier l'électronique miniaturisée associée. Les ionisations consécutives à l'irradiation et à la propagation de l'avalanche de Townsend créent de nouvelles espèces chimiques et modifient ainsi sa composition. Selon l'importance de ce phénomène, le gaz devra être renouvelé plus ou moins fréquemment.

La stricte équivalence au tissu, requise pour respecter les conditions d'application du théorème de la cavité de Bragg-Gray pour les CPET, n'est pas réalisable car la composition atomique des gaz ne permet pas d'obtenir les pondérations prescrites par l'International commission on radiological units (ICRU), notamment pour l'oxygène, le carbone et les métaux (Tab. I) [11]. Cependant, les proportions en hydrogène et en azote doivent être respectées au mieux pour obtenir une équivalence au tissu satisfaisante vis-à-vis des neutrons.

Une solution aux problèmes de pollution et de vieillissement du gaz consécutifs à l'irradiation, tout en respectant les conditions précédentes, consiste à utiliser un mélange comprenant de l'argon en plus des gaz habituellement utilisés dans les mélanges équivalents au tissu usuels à base de méthane $\left(\mathrm{CH}_{4} \mathrm{ET}\right)$ ou de propane $\left(\mathrm{C}_{3} \mathrm{H}_{8} \mathrm{ET}\right)$ (Tab. I).

Nous avons choisi l'argon car il permet d'augmenter le gain dans le gaz et d'améliorer la stabilité du mélange. En effet, l'énergie moyenne perdue par la particule chargée pour créer une paire d'ions $(W)$ dans l'argon est plus faible que dans le méthane, le dioxyde de carbone et l'azote, mais elle est sensiblement plus élevée que dans $\mathrm{C}_{3} \mathrm{H}_{8}$ (Tab. II). En conséquence, l'énergie moyenne d'ionisation d'un mélange $\mathrm{C}_{3} \mathrm{H}_{8} \mathrm{ET}$, pour lequel on remplace tout ou partie du dioxyde de carbone par l'argon, est plus faible que celle du mélange $\mathrm{C}_{3} \mathrm{H}_{8} \mathrm{ET}$ et le gain dans le gaz plus élevé. On notera aussi l'existence d'un effet Penning pour les mélanges $\mathrm{Ar}-\mathrm{C}_{3} \mathrm{H}_{8}$. Enfin, l'argon est un gaz rare, il ne se dégrade pas sous l'action du rayonnement et ne modifiera pas le fonctionnement du compteur au cours du temps. 
TABLEAU I

Composition moléculaire des mélanges de gaz équivalents au tissu usuels Molecular composition of usual tissue equivalent gas mixtures

\begin{tabular}{|lcc|}
\hline & $\mathrm{CH}_{4} \mathbf{E T}$ & $\mathbf{C}_{\mathbf{3}} \mathbf{H}_{\mathbf{8}} \mathbf{E T}$ \\
\hline $\mathrm{CH}_{4}$ & $40,5 \%$ & - \\
$\mathrm{N}_{2}$ & $3,5 \%$ & $3,5 \%$ \\
$\mathrm{CO}_{2}$ & $56,0 \%$ & $40,3 \%$ \\
$\mathrm{C}_{3} \mathrm{H}_{8}$ & - & $56,2 \%$ \\
\hline
\end{tabular}

Evaluons dans quelle proportion l'argon peut être ajouté tout en conservant au mélange de gaz son caractère équivalent au tissu.

Pour ce faire, on calcule pour différents mélanges de gaz le TLE des particules chargées (protons et électrons), le coefficient d'absorption massique en énergie des photons et le kerma pour les tissus par unité de fluence des neutrons. Ces calculs permettent de dégager un compromis entre un gain dans le gaz, le plus élevé possible, c'est-à-dire une concentration élevée d'argon, et le respect de l'équivalence au tissu biologique.

TABLEAU II

Energie moyenne (photons et électrons)

nécessaire pour créer une paire d'ions dans différents gaz [10]

Average energy (photons and electrons required to produce an ion pair in various gases [10]

\begin{tabular}{|lc|}
\hline Propane & $24,0 \mathrm{eV}$ \\
Azote & $34,8 \mathrm{eV}$ \\
Argon & $26,2 \mathrm{eV}$ \\
\hdashline$\quad \cdots$ & $33,0 \mathrm{eV}$ \\
\hline Dioxyde de carbone & \\
\hline
\end{tabular}

\begin{tabular}{|c|c|}
\hline Méthane & $27,3 \mathrm{eV}$ \\
\hline $\mathrm{C}_{3} \mathrm{H}_{8} \mathrm{ET}$ & $27,4 \mathrm{eV}$ \\
\hline $\mathrm{CH}_{4} \mathrm{ET}$ & $29,1 \mathrm{eV}$ \\
\hline
\end{tabular}

Nous avons utilisé la loi d'additivité suivante pour chacun des trois paramètres dosimétriques :

$$
P_{\mathrm{m}}=\sum_{i} \mathrm{f}_{i} \mathbf{P}_{i}
$$

où $P_{\mathrm{m}}$ est la valeur du paramètre pour le mélange,

$P_{i}$ est la valeur du paramètre pour le constituant $i[6,7,9,12,18]$,

$f_{i}$ est la fraction massique du constituant $i$ dans le mélange.

Cette règle de composition a été vérifiée avec un mélange connu. La différence avec les valeurs données par les tables a toujours été inférieure à $5 \%$ dans le domaine d'énergie étudié. 
On constate en premier lieu, sur les figures 2 à 5 , que les caractéristiques des mélanges équivalents au tissu usuels peuvent s'écarter d'environ $20 \%$ de celles correspondant au tissu de référence défini par l'ICRU. On peut donc considérer que, si les caractéristiques d'un mélange de gaz respectent cet intervalle, l'équivalence au tissu sera satisfaisante.

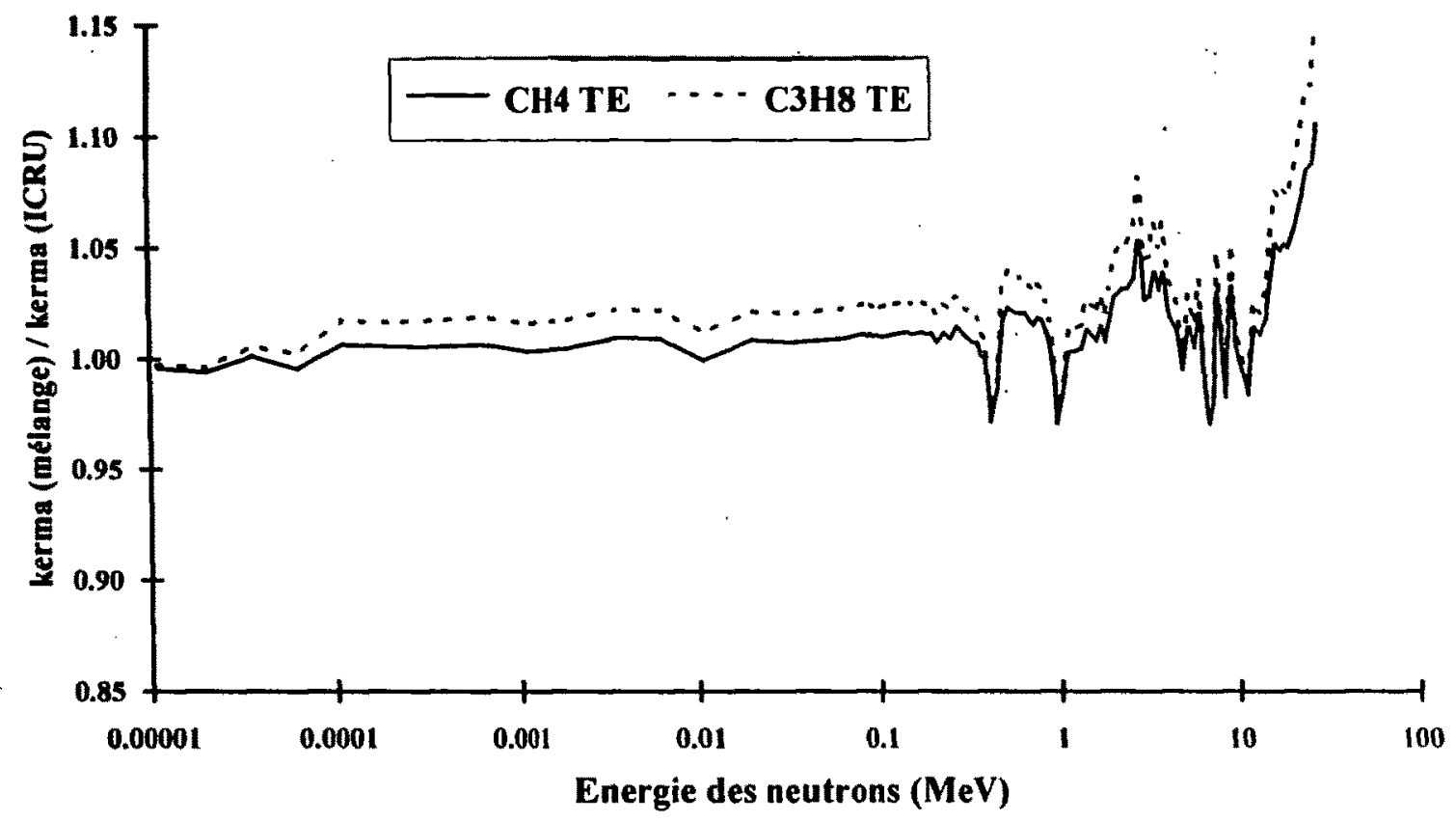

Fig. 2. - Kerma des mélanges $\mathrm{CH}_{4} \mathrm{ET}$ et $\mathrm{C}_{3} \mathrm{H}_{8}$ ET rapporté à celui du mélange équivalent au tissu défini par l'ICRU.

Kerma of $\mathrm{CH}_{4} \mathrm{TE}$ and $\mathrm{C}_{3} \mathrm{H}_{8} \mathrm{TE}$ mixture vs kerma of ICRU tissue equivalent.

La composition du mélange retenue correspond à 35,3\% d'argon, $56,2 \%$ de propane, $5 \%$ de dioxyde de carbone et $3,5 \%$ d'azote [4]. Les figures 3 à 6 présentent la réponse caractéristique de ce mélange.

Le problème le plus important est lié aux photons. En effet, on constate une grande hypersensibilité, pour les énergies comprises entre 15 et $110 \mathrm{keV}$, due à la présence d'argon. Cette hypersensibilité peut s'avérer très préjudiciable en entraînant une surestimation de la réponse aux photons et de leur facteur de qualité. Cependant, il convient de relativiser ce défaut car les interactions photon-matière qui ont lieu dans le gaz sont-beaucoup moins fréquentes que dans la cathode, comme le montre le calcul suivant.

Considérons l'énergie des photons pour laquelle l'hypersensibilité est la plus forte $(E=30 \mathrm{keV})$. Comparons l'énergie absorbée dans la cathode et dans le gaz en adoptant une géométrie plane pour simplifier les calculs. Les épaisseurs de la cathode et de la lame de gaz sont choisies en fonction des résultats des calculs du paragraphe 3. Le calcul pour la cathode tient compte du fait que le "volume efficace" (cf. § 3.3) de la cathode représente environ 1\% du volume total de celle-ci pour des photons de $30 \mathrm{keV}$. On entend par "volume efficace" le volume dans lequel les électrons, générés lors des interactions des photons, peuvent atteindre le gaz. 


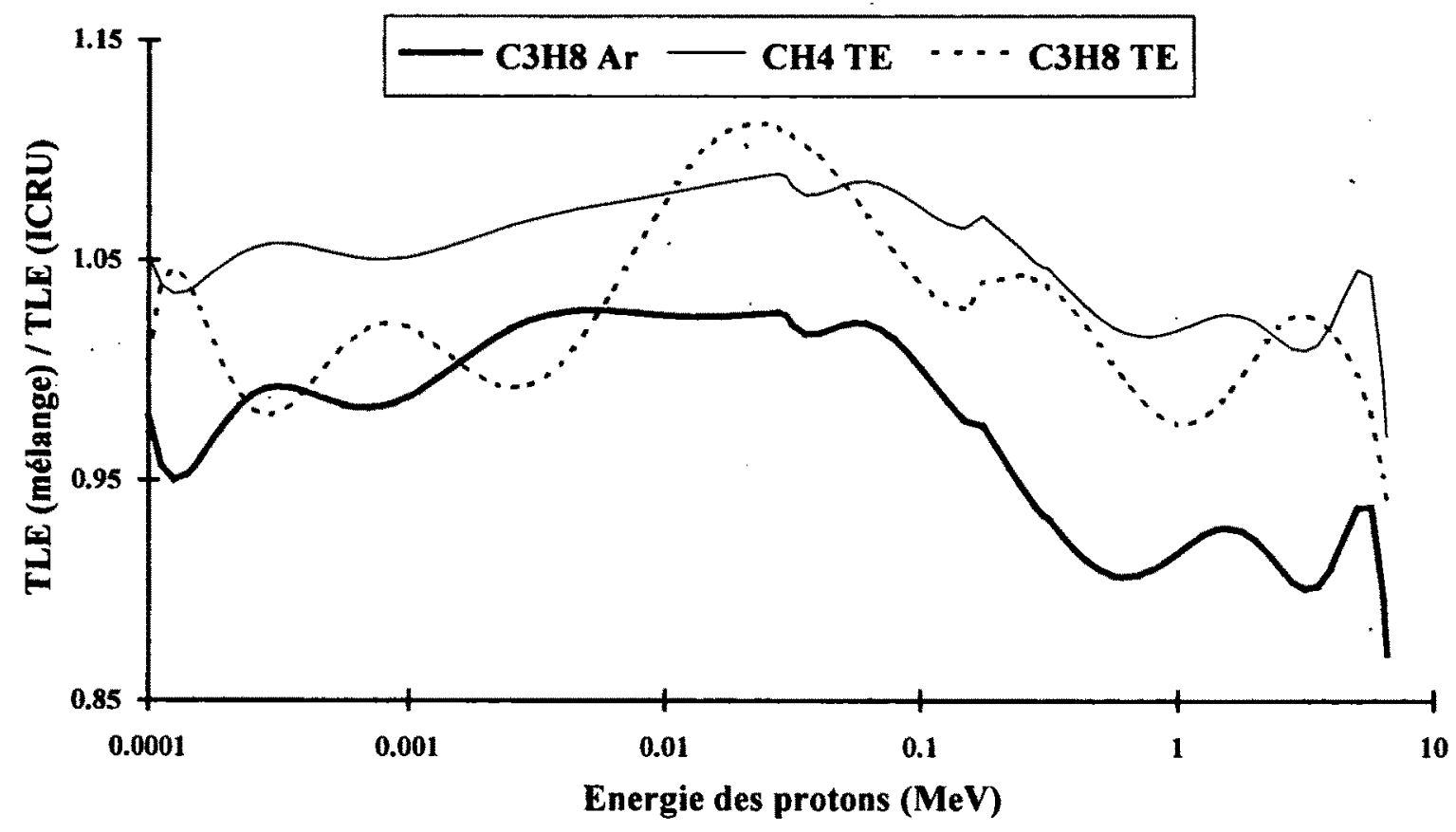

Fig. 3. - TLE des protons pour les mélanges $\mathrm{CH}_{4} E T, C_{3} \mathrm{H}_{8}$ ET et Ar- $C_{3} \mathrm{H}_{8}$ rapporté à celui du mélange équivalent au tissu défini par l'ICRU.

Proton $\mathrm{LET}$ of $\mathrm{CH}_{4} \mathrm{TE}, \mathrm{C}_{3} \mathrm{H}_{8} \mathrm{ET}$ and $\mathrm{Ar}-\mathrm{C}_{3} \mathrm{H}_{8}$ mixture vs proton $\mathrm{LET}$ of ICRU tissue equivalent mixture.

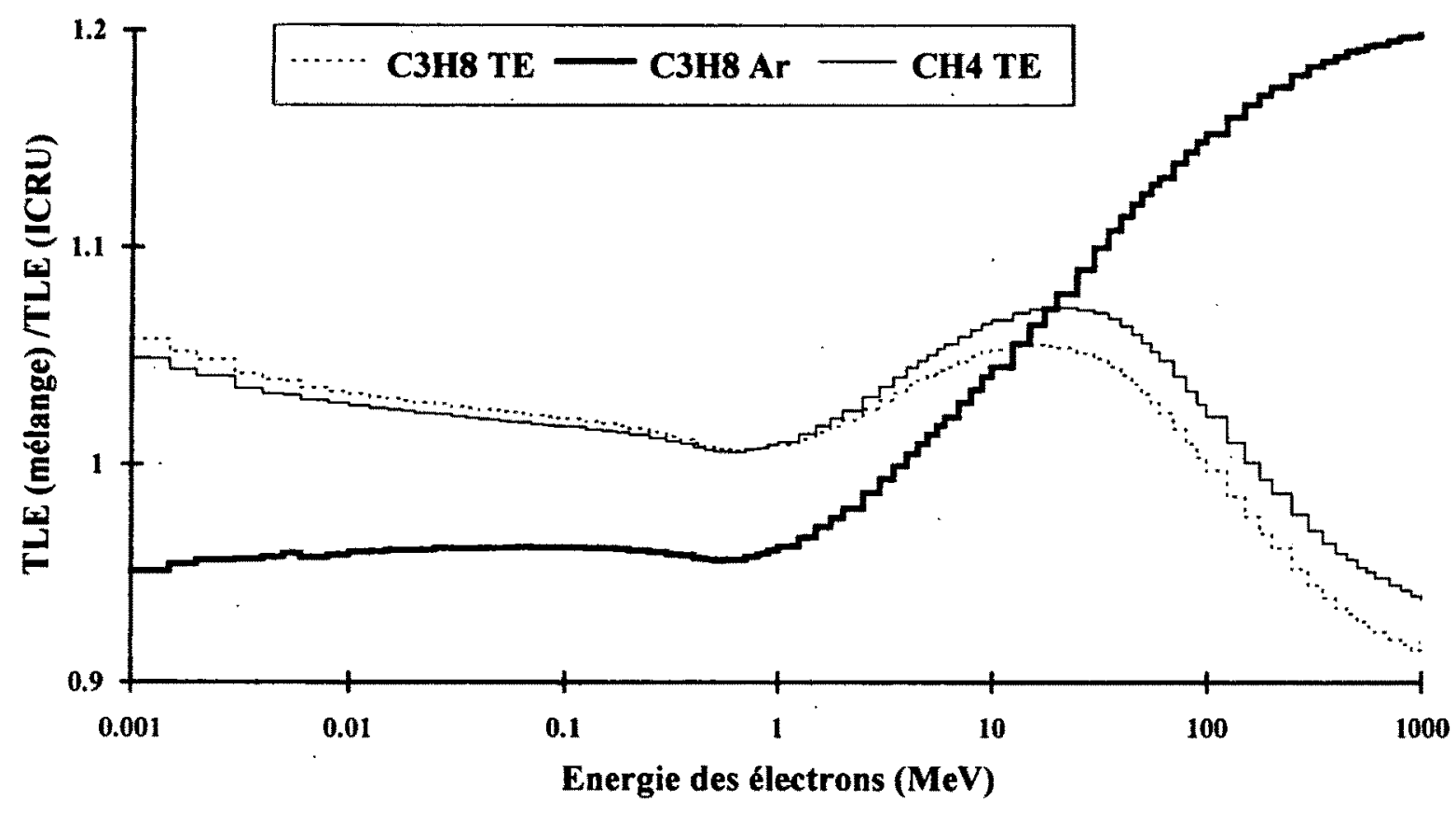

Fig. 4. - Transfert linéique d'énergie des électrons pour les mélanges $\mathrm{CH}_{4} \mathrm{ET}, \mathrm{C}_{3} \mathrm{H}_{8} \mathrm{ET}$ et $A r-C_{3} H_{8}$ rapporté à celui du mélange équivalent au tissu défini par l'ICRU.

Electron LET of $\mathrm{CH}_{4} \mathrm{TE}, \mathrm{C}_{3} \mathrm{H}_{8} \mathrm{TE}$ and $\mathrm{Ar}-\mathrm{C}_{3} \mathrm{H}_{8}$ mixture vs electron LET of ICRU tissue equivalent. 


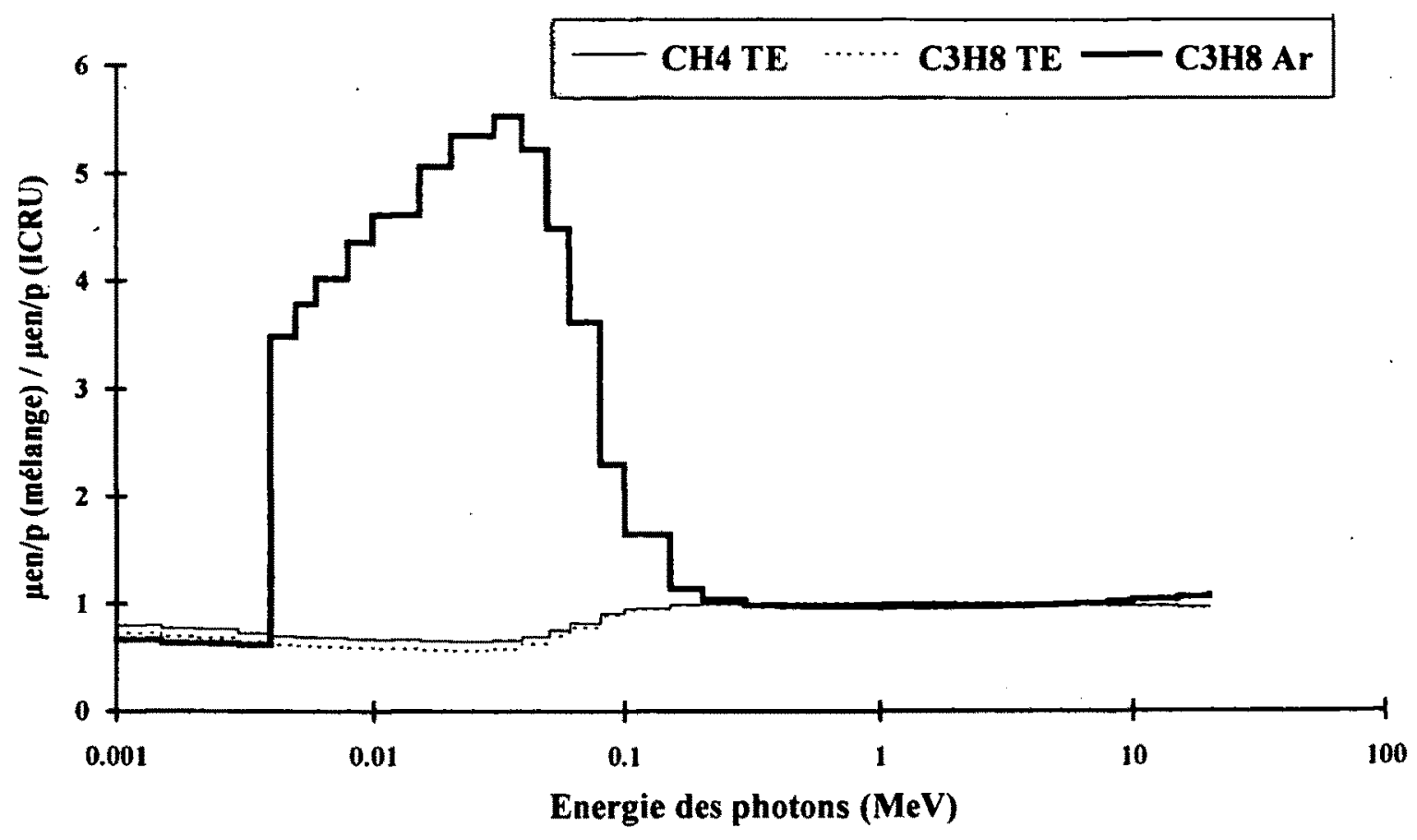

Fig. 5. - Coefficient d'absorption massique en énergie des mélanges $\mathrm{CH}_{4} \mathrm{ET}, \mathrm{C}_{3} \mathrm{H}_{8} \mathrm{ET}$ et $A r-C_{3} H_{8}$ rapporté à celui du mélange équivalent au tissu défini par l'ICRU.

Mass energy absorption coefficient ( $\mu$ en/p) of $\mathrm{CH}_{4} \mathrm{TE}, \mathrm{C}_{3} \mathrm{H}_{8} \mathrm{TE}$ and $\mathrm{Ar}-\mathrm{C}_{3} \mathrm{H}_{8}$ mixture vs $\mu e n / p$ of ICRU tissue equivalent.

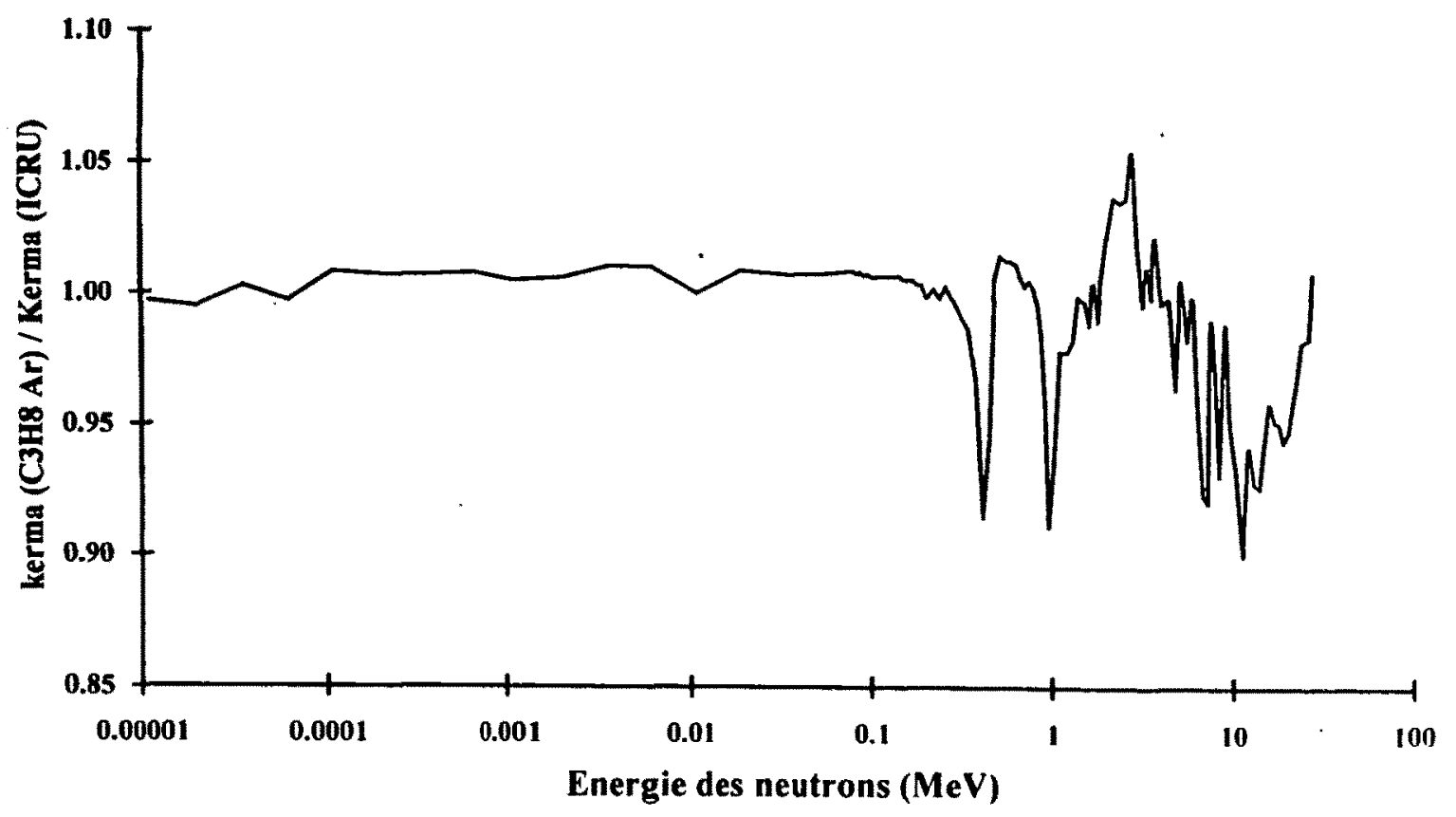

Fig. 6. - Kerma du mélange Ar- $C_{3} H_{8}$ rapporté à celui du mélange équivalent au tissu défini par l'ICRU.

Kerma of $A r-C_{3} H_{8}$ mixture vs kerma of ICRU tissue equivalent. 
EVALUATION DES CARACTÉRISTIQUES DOSIMÉTRIQUES

TABLEAU III

Absorption dans la cathode et dans le gaz Cathode and gas absorption

\begin{tabular}{|lccc|}
\hline & Cathode & Cathode efficace & Gaz ET \\
\hline Epaisseur $(\mathrm{cm})$ & 2,62 & $2,62 \times 10^{-2}$ & 2,44 \\
$\mu \mathrm{en} / \mathrm{p}\left(\mathrm{cm}^{2} / \mathrm{g}\right)$ & $1,579 \times 10^{-2}$ & $1,579 \times 10^{-2}$ & $8,407 \times 10^{-2}$ \\
$Z_{\text {eff }}$ & 2,67 & 2,67 & 3,43 \\
Densité $\left(\mathrm{g} / \mathrm{cm}^{3}\right)$ & 1,0 & 10 & $2,5 \times 10^{-4 *}$ \\
Absorption $\left(1-E / E_{0}\right)$ & $4,05 \times 10^{-2}$ & $4,13 \times 10^{-4}$ & $5,1 \times 10^{-5 *}$ \\
\hline
\end{tabular}

* Pour une pression de 100 torrs.

Les résultats du tableau III montrent que l'énergie cédée au gaz par le faisceau de photons représente $12 \%$ de l'énergie cédée à la cathode efficace. La contribution du gaz à la réponse du compteur aux photons de $30 \mathrm{keV}$ n'est cependant pas rédhibitoire. En effet, en radioprotection, les spectres de photons s'étendent bien au-delà des $2 \mathrm{MeV}$, la part des photons de $30 \mathrm{keV}$ n'y est pas prépondérante. On peut donc utiliser le mélange envisagé, malgré une sensibilité accrue pour les photons de basse énergie.

L'évaluation du gain dans le gaz est difficile car il est étroitement lié à la géométrie du compteur et à la distribution du champ électrique dans le volume sensible. Dans le CPET multicellulaire, le champ électrique n'est pas uniforme, un mode de calcul simplifié (intégration du premier coefficient de Townsend entre les points d'émission et de collection des électrons) ne donne pas de résultats cohérents. De plus, il ne tient pas compte de l'effet Penning entre l'argon et le propane. Une meilleure connaissance du gain sera obtenue par la résolution des équations décrivant le déplacement des électrons dans le gaz. Cet aspect du calcul n'a pas été abordé, il fera l'objet d'études futures en collaboration avec le CPAT. Une évaluation expérimentale a été réalisée sur un compteur cylindrique [17] (Fig. 7), elle a montré une augmentation d'un facteur 3,8 du gain dans le gaz entre les mélanges propane-argon et $\mathrm{C}_{3} \mathrm{H}_{8} \mathrm{ET}$. Les essais de vieillissement sous irradiation mettant en évidence une diminution du gain sont en cours.

\section{Evaluation théorique des caractéristiques du compteur}

\subsection{Gain de surface d'échange}

On comprend aisément que plus le nombre de canaux est important, plus la surface d'échange est grande ; une disposition en quinconce permet d'optimiser leur nombre et d'augmenter la surface d'échange par rapport au compteur de même volume. 


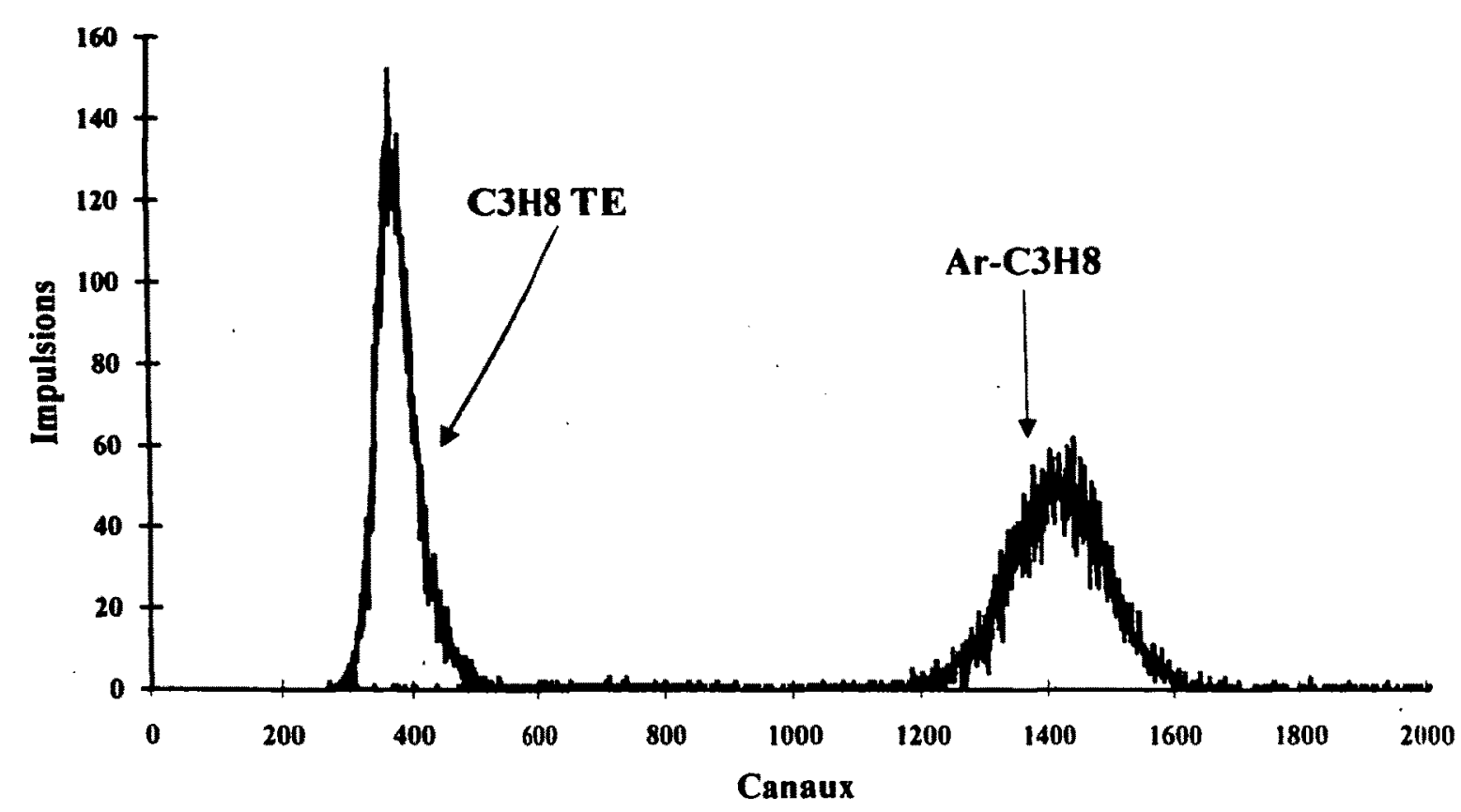

Fig. 7. - Spectres d'impulsions obtenus avec la source alpha interne d'un compteur cylindrique pour les gaz argon-propane et $\mathrm{C}_{3} \mathrm{H}_{8}$ ET.

Spectra obtained with the alpha source of a CIRCE counter filled with $\mathrm{Ar}-\mathrm{C}_{3} \mathrm{H}_{8}$ and $\mathrm{C}_{3} \mathrm{H}_{8} \mathrm{TE}$ mixture.

La figure 8 représente la variation du gain de surface d'échange entre un CPET multicellulaire et un compteur orthocylindrique de même volume extérieur, en fonction du rayon des canaux et de l'intervalle minimal entre deux canaux voisins. Dans ce diagramme, les dimensions du CPET multicellulaire demeurent constantes $(10 \times 5 \times 3,2 \mathrm{~cm})$. La profondeur des canaux est égale à $1,4 \mathrm{~cm}$.

L'optimisation du rayon des canaux et de l'intervalle minimal doit tenir compte de plusieurs contraintes : la tenue mécanique de l'ensemble, l'épaisseur minimale de matériau entre deux canaux définissant un volume dit "convexe" (cf. § 3), et le rayon de diffusion des électrons thermalisés. Ce dernier paramètre est le moins restrictif puisque, pour un champ électrique de dérive égal à $200 \mathrm{~V} / \mathrm{cm}$ dans le méthane pur sous une pression de 100 torrs, le rayon de diffusion est égal à $3,5 \times 10^{-2} \mathrm{~cm}$ pour un parcours de $1,5 \mathrm{~cm}$.

Un compromis satisfaisant est obtenu pour un rayon et un intervalle minimal entre deux canaux voisins de $0,2 \mathrm{~cm}$. Dans ces conditions, l'ensemble des deux cathodes comprend 310 canaux. Le gain de surface d'échange entre le compteur orthocylindrique, de diamètre intérieur $5 \mathrm{~cm}$, et le compteur multicellulaire est alors voisin de 5 (Fig. 8). La comparaison du CPET multicellulaire avec des compteurs sphériques ou orthocylindriques de petite taille (inférieure à $3 \mathrm{~cm}$ de diamètre), c'est-à-dire logeable dans un dosimètre comparable au radiamètre de poche, donne un gain de surface d'échange plus important (tab. IV). 


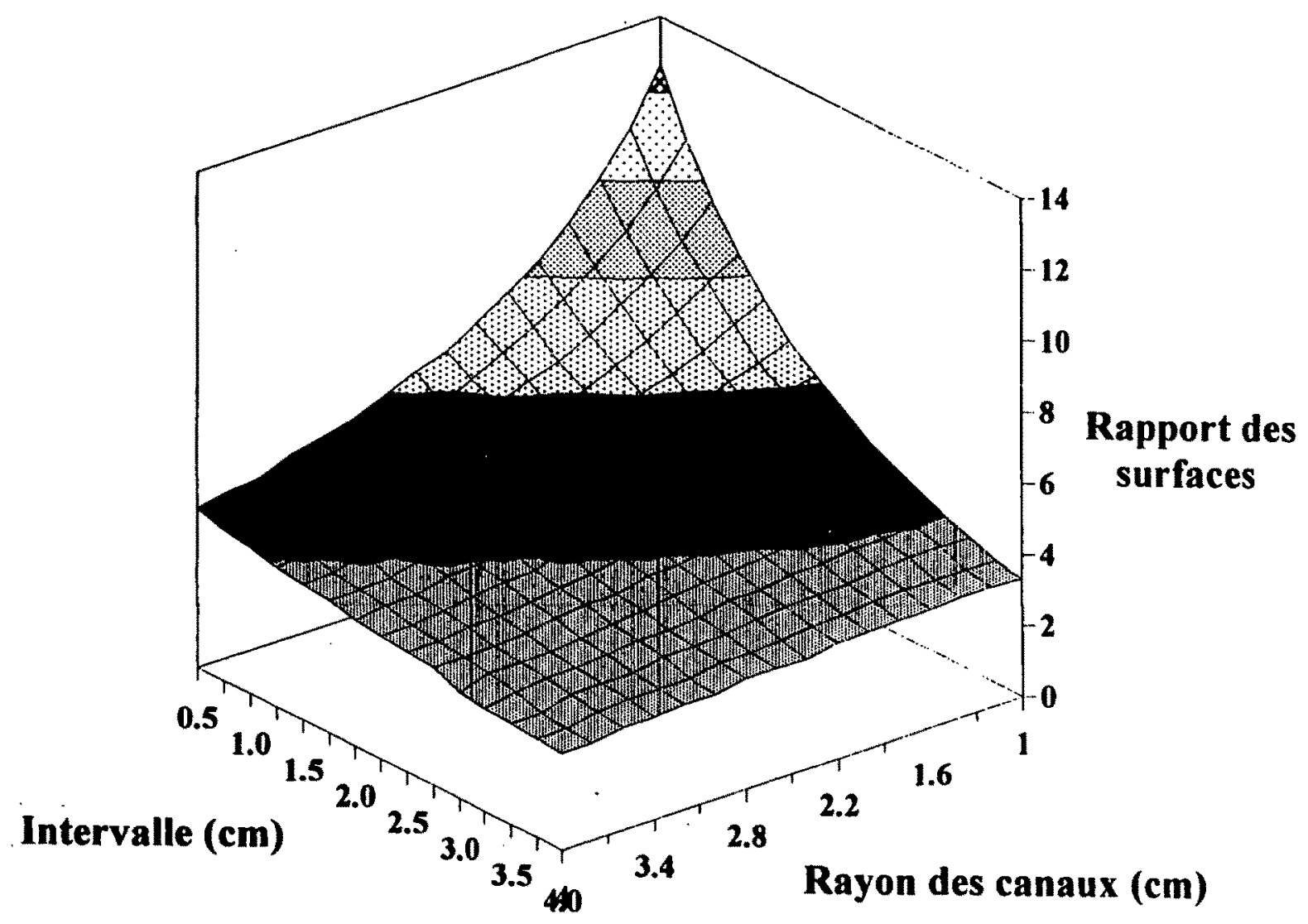

Fig. 8. - Variation du gain de surface d'échange entre le CPET multicellulaire et un compteur orthocylindrique, de même volume, en fonction du rayon des canaux et de l'intervalle minimal entre deux canaux voisins.

Surface ratio between the multicellular TEPC and an orthocylindrical counter (both having the same external volume), vs the channel radius and the distance between two nearest channel.

TABLEAU IV

Gain de surface d'échange entre le CPET multicellulaire et des compteurs sphériques ou orthocylindriques de petite taille Internal surface ratio between multicellular TEPC and small spherical or orthocylindrical counters

\begin{tabular}{|lcccccc|}
\hline $\begin{array}{l}\text { Diamètre intérieur (cm) } \\
\text { (sphère ou orthocylindre) }\end{array}$ & 0,5 & 1,0 & 1,5 & 2,0 & 2,5 & 3,0 \\
\hline $\begin{array}{l}\text { Gain de surface par rapport } \\
\text { à une sphère }\end{array}$ & 848 & 212 & 94 & 53 & 34 & 24 \\
\hdashline $\begin{array}{l}\text { Gain de surface par rapport } \\
\text { a un orthocylindre }\end{array}$ & 565 & 141 & 63 & 35 & 23 & 16 \\
\hline
\end{tabular}

Ces résultats montrent que, du seul point de vue de la sensibilité du détecteur, il est avantageux d'utiliser une structure multicellulaire [1].

La qualité de l'usinage revêt une importance particulière pour l'état de surface des parois internes du compteur et le respect des dimensions. Notamment, 
un mauvais état de surface est susceptible d'engendrer dans le gaz des microdécharges préjudiciables au fonctionnement du compteur. Il convient de noter que la texture des plastiques utilisés impose des tolérances mécaniques élevées $( \pm 0,1 \mathrm{~mm})$. Celles-ci modifient la distribution de la longueur des cordes (cf. paragraphe suivant), mais l'incertitude sur le résultat, due aux fluctuations statistiques de l'avalanche de Townsend, est supérieure à celle due aux conditions géométriques.

\subsection{Etude de la distribution de la longueur des cordes}

La corde est le paramètre géométrique qui permet de passer de la distribution des énergies "linéales" à celle des transferts linéiques d'énergie. Elle représente le parcours d'une particule chargée traversant de part en part le volume sensible. Cette définition est soumise à deux restrictions principales [2] :

- la trajectoire de la particule est supposée rectiligne,

- le volume est supposé convexe (il ne peut être traversé qu'une seule fois par la particule).

De nombreux auteurs $[17,21]$ ont assimilé la distribution de la longueur des cordes de leur compteur à une fonction de Dirac centrée sur la valeur moyenne de la distribution. Cette hypothèse simplifie les calculs dosimétriques. Dans ce cas, l'erreur commise est liée à la dispersion de la distribution.

De manière à vérifier si cette hypothèse est réaliste, nous comparons les distributions obtenues pour un orthocylindre et pour le compteur multicellulaire à l'aide d'un code de calcul utilisant la méthode de Monte-Carlo. Le principe du code est le suivant : les volumes étudiés (sphère, cylindre...) sont inscrits à l'intérieur d'une sphère à la surface de laquelle deux points sont choisis de manière aléatoire. Un pointeur fictif est déplacé le long de la droite joignant ces deux points. A chaque déplacement, le programme vérifie si le pointeur entre ou sort du volume d'intérêt. Les coordonnées des extrémités de chaque corde sont ainsi repérées. Ces opérations sont répétées jusqu'à ce que la distribution de la longueur des cordes soit statistiquement représentative de la réalité.

Les résultats obtenus avec ce code ont été comparés aux prévisions théoriques (théorème de Cauchy) pour des volumes sphériques, cylindriques et parallélépipédiques ; la différence entre les deux évaluations est toujours inférieure à $0,1 \%$.

Le volume du compteur multicellulaire est assimilé à un ensemble de "sousvolumes" constitué d'un parallélépipède et de 310 cylindres identiques. Dans un premier temps, on calcule la distribution de la longueur des cordes dans chacun des deux volumes, puis on les associe pour reconstituer le compteur multicellulaire. Lors de cette dernière étape, les différentes distributions sont pondérées par la surface totale respective des cylindres et du parallélépipède constituant le volume sensible du compteur. 
La méthode de pondération a été vérifiée sur un volume non convexe simple comprenant un cylindre et un parallélépipède (Fig. 9); l'écart entre les résultats obtenus avec le code global et avec la pondération est inférieur à $0,1 \%$.

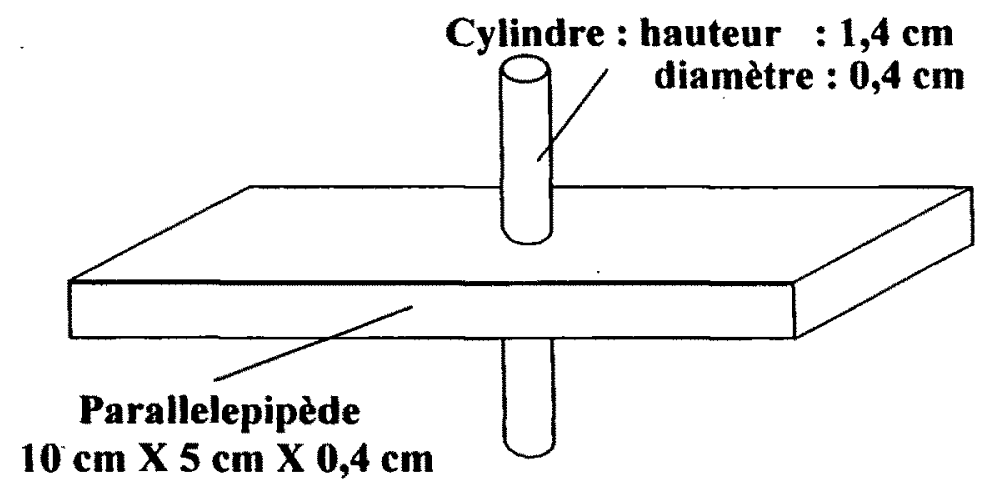

Fig. 9. - Représentation du volume sensible réduit.

Representation of the reduced sensible volume.

L'écart-type relatif sur la distribution de la longueur des cordes pour le compteur orthocylindrique est supérieure à celle du compteur multicellulaire (Fig. 10). L'erreur commise, en assimilant cette distribution à une fonction de Dirac, devrait donc être moins importante ; mais nous constatons l'existence de cordes très longues liées aux dimensions du volume parallélépipédique. Ces dernières peuvent conduire à une surestimation de l'énergie linéale si les calculs dosimétriques sont réalisés en se référant à la valeur moyenne de la distribution. La corde la plus probable coïncide avec la corde moyenne ; ainsi, même si le nombre d'événements enregistrés est faible, l'erreur commise sur le résultat dosimétrique est statistiquement peu élevée.

Ces hypothèses devront être vérifiées expérimentalement, car nos calculs ne tiennent pas compte des particules créées ou s'arrêtant dans le gaz; dans ces cas, la longueur de la corde effective est plus courte que celle de la corde géométrique, ce qui entraîne une sous-estimation de l'énergie linéale.

\subsection{Estimation du volume simulé}

Par définition, le volume simulé (voisin du micromètre cube pour une mesure correcte de l'énergie linéale) est celui d'une sphère de tissu biologique qui reçoit la même dose absorbée que le volume sensible du compteur.

Dans le cas des compteurs classiques sphériques ou cylindriques, le volume total du gaz est pris en compte pour déterminer le volume simulé. Par contre, pour le compteur multicellulaire, la géométrie du volume sensible n'est pas 
convexe. Avant de calculer le volume simulé, il faut déterminer dans quelle mesure ce volume est assimilable à un volume convexe. Pour ce faire, il faut tenir compte de deux probabilités, d'une part celle des neutrons et des photons d'interagir plusieurs fois dans le compteur, d'autre part celle des particules secondaires (électrons et noyaux de recul) de traverser plusieurs canaux.

La probabilité d'interaction multiple d'un neutron ou d'un photon dans la cathode augmente si l'énergie initiale du neutron ou du photon diminue, c'està-dire si le libre parcours moyen diminue. Pour estimer la probabilité d'interaction multiple du rayonnement, il faut donc considérer deux paramètres : l'épaisseur moyenne traversée dans la cathode et la distance moyenne entre deux canaux voisins:

- l'épaisseur moyenne est de $2,62 \mathrm{~cm}$; cette valeur est calculée en pondérant les épaisseurs traversées selon les trois directions normales aux faces du compteur par la surface de leur section droite respective;

- la distance moyenne entre deux canaux voisins est de $5 \mathrm{~mm}$, elle a été calculée dans un plan perpendiculaire à l'axe des canaux ; ce mode de calcul minimise le résultat par rapport à la réalité à trois dimensions.

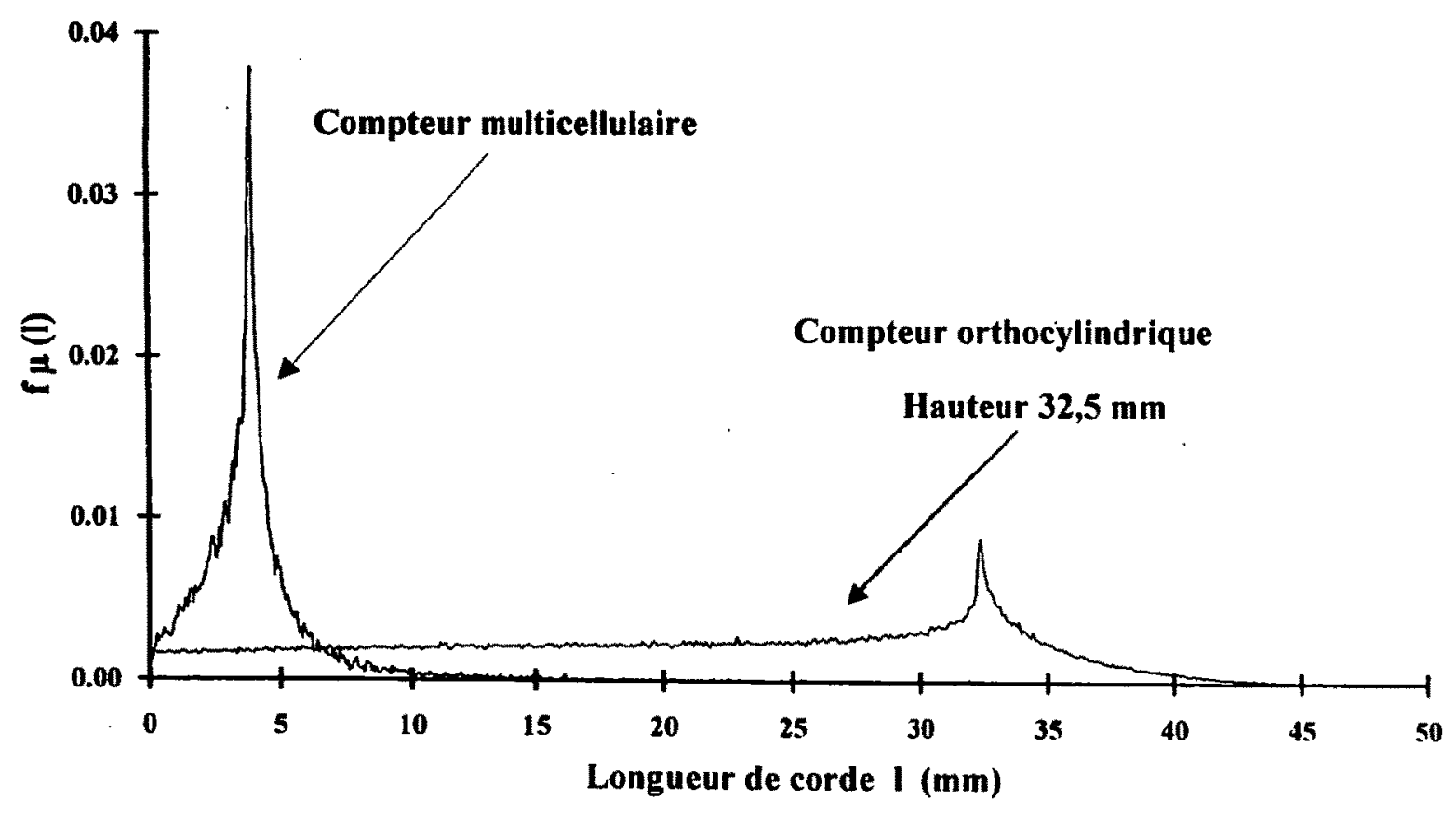

Fig. 10. - Distribution de la longueur des cordes dans un compteur orthocylindrique et dans le compteur multicellulaire.

Chord length distribution in an orthocylindrical counter and in the multicellular counter.

\subsubsection{Cas des neutrons}

En radioprotection, on considère que l'énergie maximale des neutrons est de $14,7 \mathrm{MeV}$. Compte tenu du parcours maximal des protons issus de la diffusion élastique des neutrons sur l'hydrogène, seules les interactions ayant lieu à 
proximité de la surface d'échange, c'est-à-dire dans un volume représentant moins de $1 \%$ du volume total de la cathode, sont susceptibles de produire un proton pénétrant dans le gaz. En considérant que la densité d'interaction est homogène dans toute la cathode (l'atténuation du faisceau de neutrons est considérée comme négligeable), la probabilité d'obtenir une seconde impulsion issue d'un même neutron est inférieure à $0,01 \%$. Cet effet est donc négligé.

Notons ainsi que, compte tenu de l'épaisseur minimale de matière entre deux canaux voisins, les noyaux de recul et les électrons libérés dans le gaz ne peuvent traverser qu'un seul canal. Ainsi, un même noyau de recul ne donnera pas lieu à deux impulsions.

\subsubsection{Cas des photons}

Nous sommes amenés à considérer deux catégories de photons selon que leur énergie est inférieure ou supérieure à $30 \mathrm{keV}$.

Si l'énergie est supérieure à $30 \mathrm{keV}$, le libre parcours moyen est supérieur à $2,62 \mathrm{~cm}$, donc en moyenne les photons n'interagissent qu'une seule fois dans la cathode.

Si l'énergie est inférieure à $30 \mathrm{keV}$, le libre parcours moyen est inférieur à l'épaisseur moyenne traversée dans la cathode. Ces photons sont donc susceptibles d'interagir plusieurs fois dans le compteur. Cependant, comme pour les neutrons, compte tenu du parcours maximal des électrons générés par ces photons, le volume "utile" de la cathode représente moins de $1 \%$ de son volume total. La probabilité d'observer deux impulsions distinctes issues d'un même photon est alors inférieure à $0,01 \%$ et peut donc être négligée.

Compte tenu de la distance moyenne entre deux canaux voisins, les électrons d'énergie supérieure à $1 \mathrm{MeV}$ pourront ioniser le gaz dans deux canaux voisins. Le temps de transfert entre les deux canaux est suffisamment court pour que les interactions donnent lieu à une seule impulsion, les anodes étant connectées entre elles. Ces hypothèses devront être confirmées en utilisant les codes de calcul MCNP et EG24.

Toutefois, nous pouvons estimer que, en première approximation et malgré sa forme, le volume sensible du CPET multicellulaire peut être assimilé à un volume convexe ; cela en le considérant dans son intégralité ou en le réduisant au volume de la figure 9 . Nous avons calculé le volume simulé de ce compteur en utilisant les critères proposés par Kellerer [13-15] pour des volumes convexes.

Le tableau $\mathrm{V}$ donne les volumes simulés, avec le mélange de gaz argon-propane défini précédemment et sous une pression de 100 torrs. Nous avons considéré trois situations : le volume sensible du compteur multicellulaire dans son intégralité, le volume de la figure 9, une cellule (cylindre d'élongation 8). Dans cette dernière configuration, nous négligeons le parallélépipède, c'est-à-dire que les impulsions liées à sa traversée ne sont pas prises en compte dans le 
calcul dosimétrique. Cette hypothèse correspond à une situation ou l'on réaliserait une détection de coïncidence entre les différentes anodes.

TABLEAU V

Volume simulé en fonction de la géométrie étudiée Simulated volume for the geometry studied

\begin{tabular}{|lc|}
\hline & Volume símiée \\
\hline Volume intégral & $1,3 \mu \mathrm{m}$ \\
\hline Volume fig. 9 & $3,2 \mu \mathrm{m}$ \\
\hline Volume d'une cellule & $1,6 \mu \mathrm{m}$ \\
\hline
\end{tabular}

Ces diamètres sont du même ordre que ceux des autres compteurs microdosimétriques $[8,16]$, ils permettent une mesure correcte de l'énergie linéale.

\section{Conclusion}

L'évaluation théorique des caractéristiques du compteur multicellulaire a permis de déterminer les dimensions géométriques et d'évaluer les performances du prototype de compteur multicellulaire. Ainsi, la sensibilité attendue doit être compatible avec l'utilisation du capteur en radioprotection. L'étude de la distribution de la longueur des cordes montre la possibilité d'utiliser une fonction de Dirac centrée sur la valeur moyenne de la distribution dans les calculs dosimétriques. Enfin, la dimension du volume simulé est compatible avec la méthode microdosimétrique.

Un premier prototype simplifié du CPET multicellulaire fonctionne. L'obtention de spectres microdosimétriques à partir de faisceaux de neutrons a permis de valider expérimentalement les principes de conception et de fonctionnement de l'appareil. Ces spectres ont été présentés lors du $11^{\mathrm{e}}$ symposium sur la microdosimétrie qui s'est tenu à Gatlinburg en 1992 [5]. Un nouveau compteur plus élaboré est en cours de réalisation.

Remerciements : Les auteurs expriment leurs remerciements à $M$. Nguyen Van Dat pour le prêt d'un de ces compteurs proportionnels ainsi qu'à M. Pascal Pihet pour les discussions très fructueuses concernant les principes généraux de la microdosimétrie.

Cette étude est partiellement financée par le contrat CCE numéro F13PCT93.0072. 


\section{RÉFÉRENCES}

[1] BARTHE J., MOURGUES M., BORDY J.M., BOUTRUCHE B., LAHAYE T., PORTAL G. - Etude de compteurs miniatures pour la dosimétrie individuelle des neutrons. In : "IRPA'8", Montréal, 17-22 mai 1992. Montréal : IRPA, 1992, I, 479-482.

[2] BIRKOFF R.D., TURNER J.E., ANDERSON V.E., FEOLA J.M., HAMM R.N. The determination of LET spectra from energy-proportional pulse-height measurements. I. Track-length distribution in cavities. Health Phys., 1970, 18, 1-14.

[3] BLANC D., SEGUR P., BARTHE J., BORDY J.M. - Les compteurs propotionnels à milieu équivalent au tissu en radioprotection. In : "IRPA'8", Montréal, 17-22 mai 1992. Montréal : IRPA, $1992,1,459-462$.

[4] BORDY J.M. - Contribution à la réalisation d'un compteur proportionnel à dérive équivalent au tissu destiné à la dosimétrie individuelle en radioprotection. Mémoire CNAM, Paris et Rapport CEA-R-5603, 1992.

[5] BOUTRUCHE B., BORDY J.M., BARTHE J., SEGUR P., PORTAL G. - New concept of high sensitive tissue equivalent propotional counter for individual neutron dosimetry. In : "11th Symposium on microdosimetry", Gatlinburg, Tennessee (USA), Septembre 1992. Radiat. Prot. Dosim. (à paraître).

[6] CASWELL R.S., COYNE J.J., RANDOLPH M.L. - Kerma factors for neutron energy below $30 \mathrm{MeV}$. Int. J. Appl. Radiat. Isot., 1982, 33, 1227-1262.

[7] DENNIS J.A. - Energy loss characterisitcs of heavy ions in nitrogen, carbon dioxide, argon, hydrocarbon gases and tradescantia tissue. Harwell : AERE Memorandum 2346, 1971.

[8] DIETZE G., GULDBAKKE S., KLUGE H., SCHMITZ T. - Intercomparison of radiation protection instruments based on microdosimetric principles. Braunschweig : Physikalisch technische Bundesanstalt, ND-29, 1986.

[9] HUBBELL J.H. - Photon mass attenuation and energy-absorption coefficients from $1 \mathrm{keV}$ to $20 \mathrm{MeV}$. Int. J. Appl. Radiat. Isot., 1982, 33, 1269-1290.

[10] INTERNATIONAL COMMISSION ON RADIATION UNITS AND MEASUREMENT (ICRU) - Average energy required to produce an ion pair (ICRU Report 31). Besthesda : ICRU, 1979.

[11] INTERNATIONAL COMMISSION ON RADIATION UNITS AND MEASUREMENT (ICRU) - Microdosimetry (ICRU Report 36). Bestheda : ICRU, 1983.

[12] INTERNATIONAL COMMISSION ON RADIATION UNITS AND MEASUREMENT (ICRU) - Stopping powers for electrons and positrons (ICRU Report 37). Besthesda : ICRU, 1984.

[13] KELLERER A.M. - Considerations on the random transversal of convex bodies and solutions for general cylinders. Radiat. Res. 1971, 47, 359-376.

[14] KELLERER A.M. - Proximity functions for general right cylinders. Radiat. Res. 1981, 86, 264-276.

[15] KELLERER A.M. - Criteria for the equivalence of spherical and cylindrical proportional counters in microdosimetry. Radiat. Res. 1981, 86, 277-286.

[16] MENZEL H.G., LINDBORG L., SCHIMTZ Th., SCHUHMACHER H., WALKER A.J. - Intercomparison of dose equivalent meters based on microdosimetric techniques : detailed analysis and conclusions. Radiat. Prot. Dosim., 1989, 29 (1/2), 55-68.

[17] NGUYEN V.D., LUCCIONI C., PARMENTIER N. - Average quality factor and dose equivalent meter based on microdosimetry techniques. Radiat. Prot. Dosim., $1985,10(1 / 4), 277-282$. 
[18] OLDENBURG U., BOOZ J. - Mass stopping power and pathlength of neutron produced recoils in tissue equivalent materials, I. Neutron energy $\leq 6 \mathrm{MeV}$. Rapport EUR-4786e, 1972.

[19] ROSSI H.H. - Energy distribution in absorption of radiation. Adv. Biol. Med. Phys., 1967, 11, 27-84.

[20] SAULI F. - Principles of operation of multiwire propotional and drift chambers. Rapport CERN-77-09, 1977.

[21] TINELLI P. - Etude et réalisation d'un déctecteur microdosimétrique destiné à la radioprotection. Thèse Université Paul Sabatier, Toulouse, 21 novembre 1986. 